\title{
Modelling visco-acoustic wave equation in frequency domain using mixed-grid Finite-Difference method and attenuation-dispersion model defined for quality factor
}

\author{
S.K. Avendaño*, M.A. Ospina, J.C. Muñoz-Cuartas,H. Montegranario(Group of Tomography and Inversion /UdeA)
}

Copyright 2016, SBGf - Sociedade Brasileira de Geofísica

Este texto foi preparado para a apresentação no VII Simpósio Brasileiro de Geofísica, Ouro Preto, 25 a 27 de outubro de 2016. Seu conteúdo foi revisado pelo Comitê Técnico do VII SimBGf, mas não necessariamente representa a opinião da SBGf ou de seus associados. É proibida a reprodução total ou parcial deste material para propósitos comerciais sem prévia autorização da SBGf.

\begin{abstract}
Seismic modeling is an important step in the process used for imaging earth subsurface. Current applications require accurate models associated with solutions of the equation of wave propagation in realistic medium. In this work, we propose a modelling for 2D wave propagation in a visco-acoustic medium with variable velocity and density, handled in the frequency domain under conditions that describe dissipation depending on the quality factor $Q$. We use mixed-grid finite-difference method and optimize it for the case of the visco-acoustic medium with the aim to minimize numerical dispersion. We present solutions for test cases in homogeneous and heterogeneous media and compare the analytic solutions. Further, we compare the solution using conventional grid (5-point scheme) and our mixed grid implementation (9-point scheme), finding a better response with the mixed grid 9-point scheme. We also studied the characteristics of the numerical solution, wave fields P-waves are discussed for variable velocity, density, damping functions and $Q$ values finding that the method performs very well with very much potential in applications of FWI.
\end{abstract}

\section{Introduction}

Modelling wave propagation in real sub-surface is a complex task, physics is complicated and several simplifying approximations have to be made. However in order to get precise information about the physical properties of the sub-surface, the better we model the propagation of waves, the better the result of the understanding of the properties of the medium.

Although the acoustic approach has been very successful so far in the modelling of wave propagation in the subsurface, Earth is not totally elastic neither totally viscous and a combination of both extreme behaviours should fit the properties of wave propagation. The visco-acoustic media can be defined as a medium without cross propagation but exhibits attenuation in the amplitude of the longitudinal wave, this media presents two phenomena, dissipation, produced by energy absorption such that amplitude wave is reduce especially in high frequency and dispersion, by the change in the density, where the wave velocity depends the frequency [15].

To describe the attenuation of seismic wave front energy [1] proposed a model based in linear solid material rheology and memory variables. In [5] they used a visco-acoustic wave equation to compensate for the energy decrease of wave propagation in a realistic media using an extrapolator based in the propagator of the wave equation in the forward and backward direction. While [16] present a detailed comparison of different visco-acoustic wave equations and studied their dissipation and dispersion properties, providing a rough idea about how the most commonly used viscoacoustic wave equations perform.

In particular, the realistic modelling of wave propagation is an important step in the process used for imaging earth subsurface. Currently, one of the most powerful techniques of seismic tomography that uses solutions of the wave equation is called Full Waveform Inversion (FWI) [4]. FWI consists on obtaining the model parameters of the subsurface $\hat{\mathbf{m}}$ through an inversion process that relates comparison between models $\mathbf{d}_{c a l c}(\mathbf{m})$ and observed data $\mathbf{d}_{o b s}$, minimizing the objective function $\left\|\mathbf{d}_{o b s}-\mathbf{d}_{\text {calc }}(\mathbf{m})\right\|^{2}$. To understand and emulate the complex wave phenomena in a realistic heterogeneous medium it is necessary a seismic modelling where the result is a wavefield obtained through the numerical solution of the wave equation.

We aim to provide a model for the propagation of waves in a general visco-acoustic media in the frequency domain optimized for applications in FWI (seismic tomography), considering the effects of attenuation. It is one more solution to visco-acoustic wave equation but this time in frequency domain. In other works, there are several ways to include the effects of attenuation in the wave motion equations and different models using some special mathematical tools as it is shown in [16], one of them is working in the frequency domain using a term known as complex bulk modulus, which is a method easy to implement and to relate to the quality factor $Q$ [1] and others works using Laplace transform for the model [7] and FWI [13].

This work focuses on the propagation of mechanical waves in a medium with explicit attenuation modelled through the damping functions and quality $Q$ factor. Frequency domain is adopted in this work since it always the study of effects of the modelling (attenuation, dispersion) on individual wave frequencies. We pay especial attention to the quality of the solution and provide results of the implementation of optimal mixed-grid scheme looking for minimization of the numerical dispersion in the implementation. Instead mod- 
elling explicitly the elastic and viscous excitation modes [1] we model the dissipative effect of the medium explicitly through damping functions that attenuate the wave amplitude according to the quality factor of the media for different frequencies.

\section{Equation of propagation in medium visco- acoustic, mathematical and physical formulation}

Wave propagation rests on the basis of mechanics of the continuum medium. Basic equations that describe the motion of the media and perturbations propagating inside it are the conservation of mass, linear and angular momentum conservation, and for closure one requires an equation of state or governing equation that relates the intrinsic properties of the media with its dynamical variables [10]

Now, if we assume that the medium is visco-acoustic, irrotational and compressible but assuming that the flow is approximately constant we can reduce the governing equation to:

$$
\sigma_{i j}(\mathbf{x}, t)=-p(\mathbf{x}, t) \delta_{i j}=\nabla \cdot \mathbf{u}(\mathbf{x}, t) \circ M(\mathbf{x}, t) \delta_{i j}
$$

Where $p(\mathbf{x}, t)$ is a pressure wave and $\delta_{i j}$ is a kronecker delta. For an acoustic medium the wave propagation is longitudinal, such that oscillation of the particles is parallel to the direction of wave propagation then, there is no shear waves. On the other hand for a visco-acoustic medium pressure wave is related with $\mathbf{u}(\mathbf{x}, t)$, the particle displacement, and $M(\mathbf{x}, t)$ is the time derivative of the elastic tensor often named complex bulk module [4]. $M(\mathrm{x}, t)$ accounts for the elastic and dissipative properties of the media.

Equations (1) is a Hooke's law and combining states the conservation of mass, linear and angular momentum conservation one can get Newton's law.

$$
\left(\frac{\partial}{\partial t}+\gamma(\mathbf{x})\right) \mathbf{v}=\frac{1}{\rho(\mathbf{x})}(\nabla \cdot \boldsymbol{\sigma}+\mathbf{f})
$$

Where $\gamma(\mathbf{x})$ is called the rate deformation function, such that it represents the temporal variation of the stress tensor and is related to the viscosity of the medium, $\rho$ is the density, $\mathbf{v}$ represents the particle velocity, $\sigma$ is the stress tensor, $\mathbf{f}$ represents the body forces. It is noticeable that this balance of force in the equation (2) is similar to the balance of forces for damped harmonic oscillator, where $\gamma(\mathbf{x})$ it is an important term in the relation of energy stored and energy lost per cycle [15]. Applying a Fourier transform to eqs. (1) and (2) we have

$$
\begin{gathered}
-i \omega P(\omega, \mathbf{x})=M(\omega, \mathbf{x}) \nabla \cdot \mathbf{V}(\omega, \mathbf{x}) \\
-i \omega \mathbf{V}(\omega, \mathbf{x})=-\frac{b(\mathbf{x})}{\xi(\omega, \mathbf{x})} \nabla P(\omega, \mathbf{x})+\frac{b(\mathbf{x})}{\xi(\omega, \mathbf{x})} \mathbf{F}(\omega, \mathbf{x})
\end{gathered}
$$

Where $\xi(\omega, \mathbf{x})=1+i \frac{\gamma(\mathbf{x})}{\omega}$ and $b(\mathbf{x})=\frac{1}{\rho(\mathbf{x})}$. The propagation of waves in a visco-acoustic media presents both dissipation and dispersion. These effects are modeled using the damping functions, $\xi(\omega, \mathbf{x})$ so that the attenuation occurs, and since the realistic situation implies dispersivity in the media, this damping must be modelled as a frequency dependant process.
The complex bulk modulus must be related to $\xi(\omega, \mathbf{x})$ subject to the constraint that when no dissipation $M(\omega, \mathbf{x}) \rightarrow$ $K(\omega, \mathbf{x})$, where $K(\omega, \mathbf{x})$ is an acoustic bulk modulus, so we define

$$
M(\omega, \mathbf{x})=K(\mathbf{x}) \alpha(\omega, \mathbf{x})=K(\mathbf{x}) \frac{1}{\xi(\omega, \mathbf{x})}
$$

Where $\alpha(\omega, \mathbf{x})$ is complex. To establish a relation between $\alpha$ and $\xi$ we combine the equations (3) and (4) in the homogeneous case, with $\rho=$ cte, $c=$ cte and $\gamma=$ cte and obtain $\left(\frac{\xi}{\alpha} k^{2}+\nabla^{2}\right) P=\nabla \cdot \mathbf{F}$. If $\alpha=\xi$ reduces to the acoustic wave equation in frequency domain, but since $\alpha=\frac{1}{\xi}$ then the equation is recast as

$$
\left(\tilde{k}^{2}+\nabla^{2}\right) P=\nabla \cdot \mathbf{F}
$$

The equation (6) is the Helmholtz equation with complex coefficients, where $\tilde{k}$ is called complex wave number. The real part of $\tilde{k}$ is $\frac{\omega}{c}$, the conventional wave number, and the imaginary part $\frac{\gamma}{c}$ is the pseudo wave number. This concept is applied in attenuation-dispersion models related to the quality factor $Q$. In other words, we can relate our damping function $M(\omega, \mathbf{x})$ with the models of attenuation and dispersion, in this way it allows us to propose damping functions that have an actual relationship with the properties of the medium we want to model.

Then, the equation for wave propagation in our viscoacoustic media can be obtained combining equations (3), (4) and (5) as

$$
\begin{aligned}
\frac{\omega^{2} P(\omega, \mathbf{x})}{K(\mathbf{x})}+ & \frac{1}{\xi(\omega, \mathbf{x})} \nabla \cdot\left(\frac{b(\mathbf{x})}{\xi(\omega, \mathbf{x})} \nabla P(\omega, \mathbf{x})\right)= \\
& \frac{1}{\xi(\omega, \mathbf{x})} \nabla \cdot\left(\frac{b(\mathbf{x})}{\xi(\omega, \mathbf{x})} \mathbf{F}(\omega, \mathbf{x})\right)
\end{aligned}
$$

In this situation we have the freedom to choose the way we model the attenuation. For example, using the models described in [15] we decided to study three different attenuation-dispersion models, where we focus on its dependence on the frequency and dispersion associated with them.

1. Kolsky's: $\xi(\omega, \mathbf{x})=1+i \frac{1}{2 Q(\mathbf{x})}$

2. Cole-Cole: $\xi(\omega, \mathbf{x})=1+i g(Q, \mathbf{x}) \omega^{-n}$

3. General linear model: $\xi(\omega, \mathbf{x})=1+i h(Q, \mathbf{x}) \omega^{n}$

Where $g(Q, \mathbf{x})$ and $h(Q, \mathbf{x})$ are function, these can be found in the reference [15].

\section{Numerical solution technique: Mixed Grid and Lumped Mass strategy}

In general, obtaining a solution of equation (7) is not possible for arbitrary medium properties and boundaries. A natural approach is then to look for numerical solutions using e.g. finite differences. Although the finite difference method is widely used for solving differential equations, it has very well known problems of numerical dispersion that are related to the discretization of the media. 
To avoid these numerical problems [6] propose a scheme of two overlapping grids to solve the PDE. One of the grids has a conventional cartesian grid, and the second one is a rotated cartesian grid, assuming a $2 \mathrm{D}$ problem with coordinates $(x, z)$ and using (3), (4), (5) In this kind of setup, the solution is obtained evaluating the fields in a total of 9 grid points, thus reducing the numerical dispersion and trying to cover all directions of propagation. Then, using centered finite-difference for partial derivatives, in conventional grid, we find the derivatives of the function $\mathbf{V}(\mathbf{x}, \omega)$ and the derivatives of $P(\mathbf{x}, \omega)$ a function of the grid spacing $\Delta$. Now, for the rotated grid, using a rotation of $45^{\circ}$, we obtain the transformation rule for the derivatives in such system, then in the rotated system

$$
\frac{\partial}{\partial x}=\frac{\sqrt{2}}{2}\left(\frac{\partial}{\partial x^{\prime}}+\frac{\partial}{\partial z^{\prime}}\right) \quad \frac{\partial}{\partial z}=\frac{\sqrt{2}}{2}\left(-\frac{\partial}{\partial x^{\prime}}+\frac{\partial}{\partial z^{\prime}}\right)
$$

Such that centered finite-difference of partial derivatives, in rotated grid of $\mathbf{V}(\mathbf{x}, \omega)$ and and $P(\mathbf{x}, \omega)$ are found depending on the rotated coordinates, using the points on the diagonals and then combined to obtain the partial derivatives according eq. (8). Now, to improve the accuracy of the mixed-grid stencil the pressure acceleration term $\frac{\omega^{2}}{K(\mathbf{x})} P(\omega, \mathbf{x})$ may not be taken as a term implying only the collocation point (the so-called lumped approximation), the implementation of the mixed grid aims to reduce the numerical dispersion, taking 9 points to find the values of the derivatives, if the free term takes only the point value the contribution of rotated grid will be diminished and the homogeneity of the solution will be lost to some degree [12].

In order to solve this issue we may approximate this term by using a weighted average over the mixed operator stencil nodes, as it is done in finite-element modelling ( [9], [14]). Then in this situation the lumped mass term shall take the form:

$$
\begin{array}{r}
\left.\frac{\omega^{2} P(\omega, \mathbf{x})}{K(\mathbf{x})}\right|_{(i, j)}= \\
\frac{\omega^{2}}{K_{(i, j)}}\left(m_{2} P_{(i, j)}+m_{3} \Sigma_{1(i, j)}+\frac{m_{4}}{4} \Sigma_{2(i, j)}\right)
\end{array}
$$

With $m_{4}=1-m 2+4 m_{3}$, where $m_{2}$ and $m_{3}$ are coefficients regulating the relation between the contribution of the terms by the conventional and rotated grid in the lumped mass term. The other terms in the conventional grid are given by $\Gamma_{(i, j)}$ and the rotated grid $\Theta_{(i, j)}$. Then, the final version of discretization of equation (7) is

$$
m_{1} \Gamma_{(i, j)}+\left(1-m_{1}\right) \Theta_{(i, j)}+\left.\frac{\omega^{2} P(\omega, \mathbf{x})}{K(\mathbf{x})}\right|_{(i, j)}=S_{(i, j)}
$$

Where $m_{1}$ is a coefficient regulating the relation between the contribution of the terms by the conventional and rotated grid but in the remaining divergence terms in eq. (7). That after simplifications can be finally written as

$$
\begin{aligned}
D_{(i, j)} P_{(i, j)} & +D 1_{(i, j)} P_{(i+1, j)}+D 2_{(i, j)} P_{(i-1, j)}+ \\
D 3_{(i, j)} P_{(i, j+1)} & +D 4_{(i, j)} P_{(i, j-1)}+D 5_{(i, j)} P_{(i+1, j-1)}+ \\
D 6_{(i, j)} P_{(i+1, j+1)} & +D 7_{(i, j)} P_{(i-1, j+1)}+ \\
D 8_{(i, j)} P_{(i-1, j-1)} & =S_{(i, j)}
\end{aligned}
$$

As it is well known, one of the advantages of the solution of the problem of wave propagation in frequency domain is that at the end, the problem can be formulated as a linear problem for each single frequency $\omega$. In our case, that linear problem shall have the form $\mathbf{A p}=\mathbf{s}$ Where the matrix A is made of the coefficients $D_{(i, j)}$. This matrix is sparse, indefinite, complex and square. The vector $s$ is the source vector discretized around the coordinates $\left(x_{0}, z_{0}\right)$. Finding $\mathbf{p}$ as the solution to the problem stated in $\mathbf{A p}=\mathbf{s}$ is equivalent to solve the system of equations (7).

An important ingredient for the solution of equation (7) is the boundary conditions. In this work we will assume a simplistic approximation and will use perfectly-matched absorbing boundary conditions. In our case, we simply expanded the computational domain from $N x \times N z$ to $N x e \times$ $N z e$ where $N x e=N x+n_{x p m l}$ and $N z e=N z+n_{x p m l}$, where $n_{x p m l}$ and $n_{z p m l}$ are the number of extra points from the boundary condition. In the expanded region the damping functions have the form [12]

\section{Dispersion Analysis}

As it is clear from the use of finite differences, the numerical solution requires a discretization to represent the physical domain that in our case is implemented in a regular mesh which has both a finite physical extent and finite number of mesh points. However, the number of points per unit wavelength that the approximate field encounters is not the same in every direction, and this artificial artefact due to the discreteness adds to the numerical dispersion of the solution. Clearly, this is not a problem that can be ignored. Since the solution is not exact there is already an error on the approach, if we add this pollutive effect to the solution, we will have, specially at long wavelengths, an inappropriate estimate of the solution due to the undersampling where long wavelenghts are not sampled at high enough spatial frequencies to provide a trustable solution at those wavelenghts [3].

In this part we will make use of the know behaviour of the problem of wave propagation in a homogeneous media (eq.6). In this case for constant velocity, density and attenuation in a media without source we have that the wave equation (eq.6) may be written as

$$
\begin{aligned}
\left(\Delta+\tilde{k}^{2}\right) P\left(x_{i}, z_{j}\right) \approx m_{1} \Gamma_{(i, j)} P & + \\
\left(1-m_{1}\right) \Theta_{(i, j)} P+\Phi\left(m_{2}, m_{3}\right)_{(i, j)} P & =0
\end{aligned}
$$

Where $m_{1}, m_{2}, m_{3}$ are parameters that we will find subject to the condition of minimization the dispersion of the solution. To do so, remember that in this case $\rho$ and $c$ having a constant value, the problem has an analytic solution, given by $P(\mathbf{r})=P_{0} e^{-i(\tilde{\mathbf{k}} \cdot \mathbf{r})}$ Where $\tilde{\mathbf{k}}$ is a complex wave vector and $\mathbf{r}$ is the position vector. Now, If we introduce the solution into the numerical scheme in equation (11), we see that the numeric complex wavenumber can be written as the ratio of two arbitrary functions $A$ and $B$ as

$$
\tilde{k}^{2}=\frac{1}{\Delta^{2}} \frac{A\left(\tau_{r}, \tau_{i}, \theta\right)}{B\left(\tau_{r}, \tau_{i}, \theta\right)}
$$

Where if $G_{r}$ is the number of grid points per wavelength, 
then $\tau_{r}=\frac{1}{G_{r}}$ is the number of wavelengths per grid point. The same relation holds for the pseudo grid number for pseudowavelength $G_{i}$ and $\tau_{i}=\frac{1}{G_{i}}$ and where $\theta$ is the propagation angle. Taking square root of eq. (12), dividing by the theoretical estimate of $\tilde{k}, \tilde{k}^{T}$ and separating in to real and imaginary parts, our objective function is

$$
\begin{gathered}
\chi\left(m_{1}, m_{2}, m_{3}\right)=\int_{V}\left[\left(1-\frac{1}{2 \pi \tau_{r}} \Re\left(\sqrt{\frac{A}{B}}\right)^{2}\right.\right. \\
+\quad\left(1-\frac{1}{2 \pi \tau_{i}} \Im\left(\sqrt{\frac{A}{B}}\right)^{2}\right] d \theta d \tau_{r} d \tau_{i}
\end{gathered}
$$

Which we try to minimize for $\theta \in[0, \pi / 2]$ and $\tau_{r}, \tau_{i} \in$ $[0.001,0.15]$ [2]. The result of minimization given the values of $m_{1}, m_{2}$ and $m_{3}$ that minimize the dispersion, so is $m_{1}=0.6667, m_{2}=0.6556, m_{3}=0.0889$. It is important to mention that when there is no attenuation, we get the same result that [6] $m_{1}=0.5461, m_{2}=0.6248, m_{3}=0.09381$. Also using the values $m_{1}=1.0, m_{2}=1.0, m_{3}=0.0$ we can recover the usual 5-point scheme.

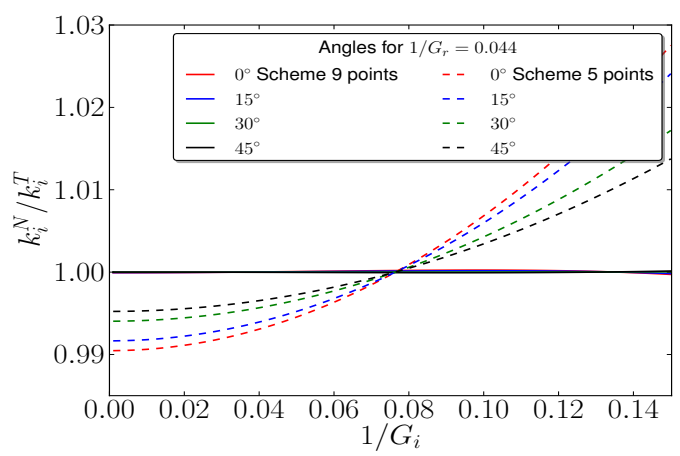

Figure 1: Ratio between the imaginary part of the theoretical and numerical wave number for Ratio $k_{i}^{N}$ to $k_{i}^{T}$ for $1 / G_{r}=0.044$

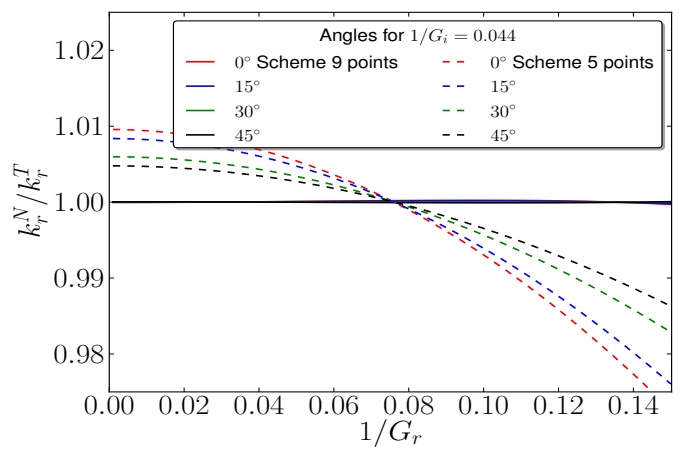

Figure 2: Ratio between the real part of theoretical and numerical wave number for Ratio $k_{r}^{N}$ and $k_{r}^{T}$ for $G_{i}=0.044$

To study the difference between the numerical dispersion produced by the scheme of 9-points and scheme 5-points, we plot in figures (1) and (2) the relation between the theoretical and numeric wave numbers for the real and imaginary parts, $\frac{k_{r}^{N}}{k_{r}^{T}}$ and $\frac{k_{i}^{N}}{k_{i}^{T}}$. We use the optimum values found in the minimization, and plot the behaviour for different propagation angles, $\theta=0, \frac{\pi}{12}, \frac{\pi}{6}, \frac{\pi}{4}$ in the eq. (12) We calculate $\frac{k_{r}^{N}}{k_{r}^{T}}$ for $\tau_{r}=0.044$ and $\tau_{i}=[0.001,0.15]$, and $\frac{k_{i}^{N}}{k_{i}^{T}}$ for $\tau_{i}=0.044$ and $\tau_{r}=[0.001,0.15]$

To understand this figure, keep in mind that according to was the closer these ratios are to 1 , the lower the numerical dispersion and better the quality of the solution. Solid lines show the ratio computed for the scheme of 9points while dashed lines show the ratio estimated for the 5 -point scheme. The different lines for each scheme (different colour lines) show the result for different propagation angles $\theta$. Notice that for a fixed value of $\tau_{r}$ or $\tau_{i}$ the dispersion is smaller for the 9-point scheme. Figures (1) and (2) clearly show that the implementation of the mixed grid and optimization presented in section 3 provides a high accuracy solution to the problem of wave propagation in a complex visco-acoustic medium at a relatively low computational cost.

\section{Constant density, velocity, and attenuation}

In frequency domain, the Helmholtz equation with source (eq 5), can be written as

$$
\tilde{k}^{2} P(\omega, \mathbf{x}, \gamma)+\nabla^{2} P(\omega, \mathbf{x}, \gamma)=S(\omega, \gamma)
$$

Where $S(\omega, \gamma)$ is a source. And an analytic solution to this equation is [2]

$$
P(\omega, \mathbf{x}, \gamma)=i \pi H_{0}^{(2)}\left(\frac{\omega-i \gamma}{c} r\right) S(\omega, \gamma)
$$

Where $r$ accounts for the distance between the source and any point with coordinates $(x, z)$ and is given by $r^{2}=$ $\left(x-x_{0}\right)^{2}+\left(z-z_{0}\right)^{2}$ With $x_{0}$ and $z_{0}$ the position of source. In order to compare the results of this analytic solution with results obtained with the 9-point and 5-point schemes, we compute the value of the $\mathrm{P}$-wave amplitude in an area of $2[\mathrm{Km}] \times 2[\mathrm{Km}]$ by placing a line of receivers at $100[\mathrm{~m}]$ and $1100[\mathrm{~m}]$, finding the waveform response at that point. The constant velocity of the media is 2100 [m/s], the Ricker frequency is $30[\mathrm{~Hz}]$, the constant quality factor is $Q=50$, using the damping function for Kolsky's Model, the cell size $\Delta$ is $\frac{\lambda}{G_{r}}$, where $G_{r}=7$, Therefore, $\tau_{r} \approx 0.14$, which is within our range of values for $\tau_{r},[0.001,0.15]$. The position of the source is $x_{0}=1[\mathrm{Km}]$ and $z_{0}=1[\mathrm{Km}]$.

In Fig. 3 we show the normalized amplitude of the P-wave as a function of the offset for shots in the receivers at $1100[\mathrm{~m}]$ deep (near the source) and difference between $\mathrm{P}$-wave found by the analytic solution and 9-point scheme and 5-point scheme, at three different frequencies. In Fig. 4 we show the normalized amplitude of the P-wave as a function of the offset for shots in the receivers at 100[m] deep (far from the source) and difference between P-wave found by the analytic solution and 9-point scheme and 5-point scheme, at different frequencies. Amplitudes are normalized as dividing by its maximum value. The solid line is the analytic solution, the dashed line is the solution for 5-point scheme and the points is a solution for 9-point scheme. It 
is worth to note that the solution obtained by the 9-point scheme is the closest to the analytic solution.

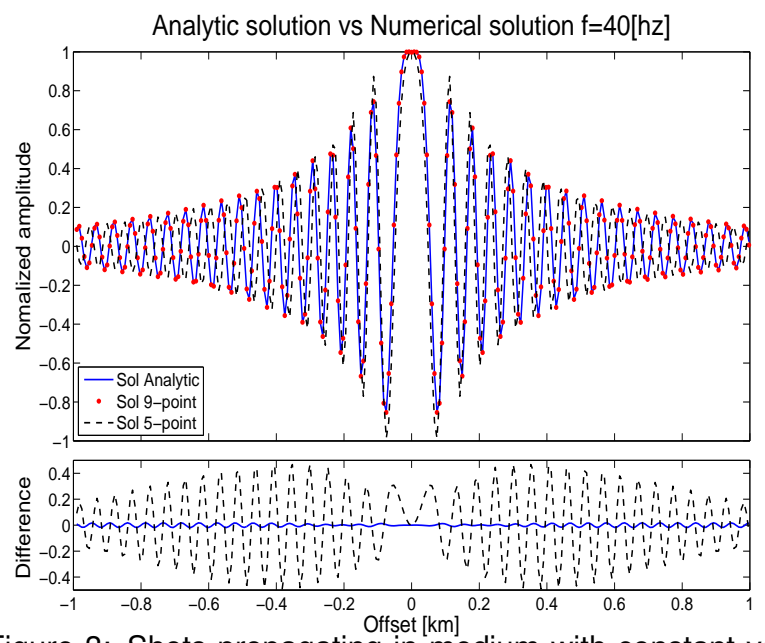

Figure 3: Shots propagating in medium with constant velocity, density and attenuation for $f=40[\mathrm{~Hz}]$ and receivers in 1100 [m] deep (close to the source)

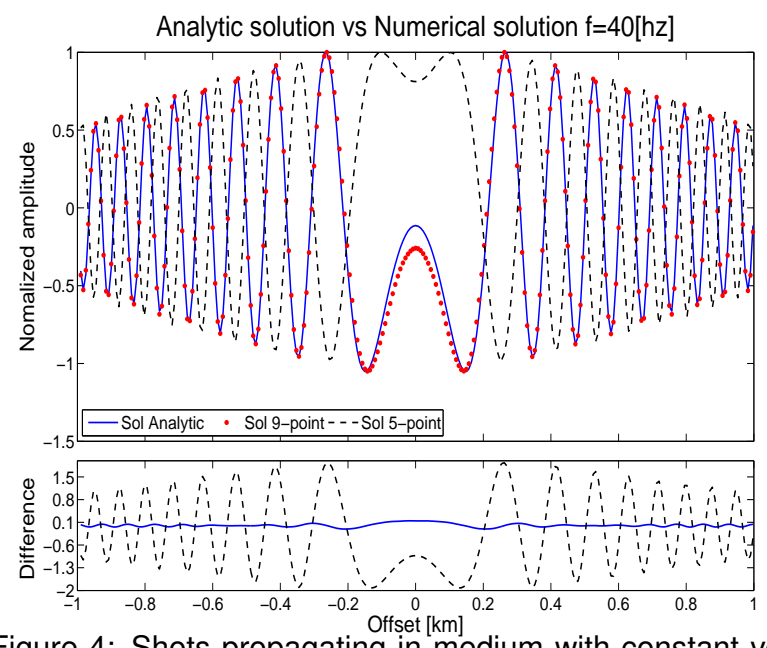

Figure 4: Shots propagating in medium with constant velocity, density and attenuation for $f=40[\mathrm{~Hz}]$ and receivers in 100 [m] deep (away from the source)

In order to avoid such dispersion in the solution for the 5point scheme, we would have to use a larger amount of points [6] and that would mean a greater computational cost. In that sense we can conclude that the scheme 9points provides an appropriate solution at an acceptable computational cost.

\section{Media with variable velocity, density and attenua- tion}

The density, in this case, was calculated following the relation [11]. In this case the values for the quality factor associated with the different values of the velocity in the multiple layer media are modelled according [8]. For this test we decided to use a complex velocity profile. As it can be seen in Figure (5), in this case we have several wedge and important discontinuities with velocity contrast ranging from $\sim 1.2$ to $\sim 3$. For this setup the source is close to the surface at $15[\mathrm{~m}]$ depth.

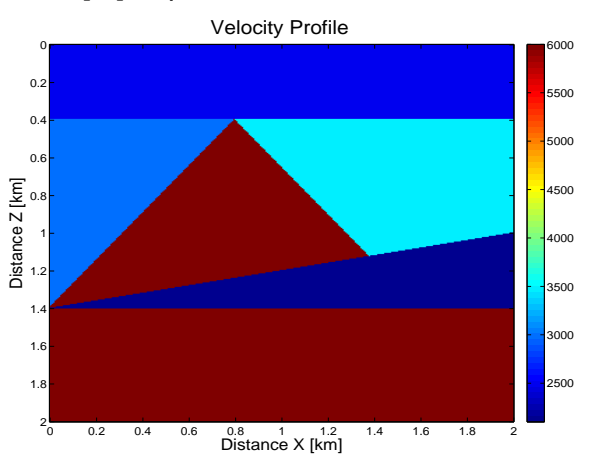

Figure 5: Geometric configuration of the velocity profile

In figures 6, 7 and 8 we show the real, imaginary part and modulus of the P-wave field for frequencies of 5,25 and 50 [Hz]. The result is less uniform than in the previous figures, revealing a natural response to the anisotropy of the velocity field. One can see how the features of the velocity field can distort the wavefront, even at large distances. Numerical dispersion have been minimized, so most of what can be seen is real response of the wave propagating inside the complex media.

\section{Conclusions and discussion}

In this work we have studied the propagation of waves in a visco-acoustic medium through explicit modelling of the attenuation making use of damping functions that allow for dispersion that depends on the quality factor $Q$ and implemented a finite difference scheme to solve the problem in frequency domain. Special care have been taken on the pollutive numerical dispersion issues of the modelling, for which we have used a mixed grid technique and optimal setup of the intercalated grids to minimize numeric dispersion.

We have shown the ability of the optimization scheme to minimize the numerical dispersion for the visco-acoustic case, and show that indeed the mixed grid scheme (9point) with the optimization scheme provides solutions that are very much close to the real solution than the solutions obtained for the classic 5-point scheme. Comparison of the numeric scheme with the analytic solution obtained for the case of wave propagation in a homogeneous medium has shown the advantages of the optimized mixed grid scheme. As it was shown in figures 3 and 4 the optimized 9-point mixed grid scheme provides solutions that are in general a few percent away from the analytic solution, while the standard 5-point scheme deviates notoriously from the analytic solution even for this simplistic case.

We have also shown that this modelling works quite well in models of high velocity/density contrast, commonly found in the field. We have shown that the explicit modelling of attenuation allows to model easily the superposition of complex velocity fields and to resolve properly the behaviour of waves in such scenarios. We have verified the behaviour of 


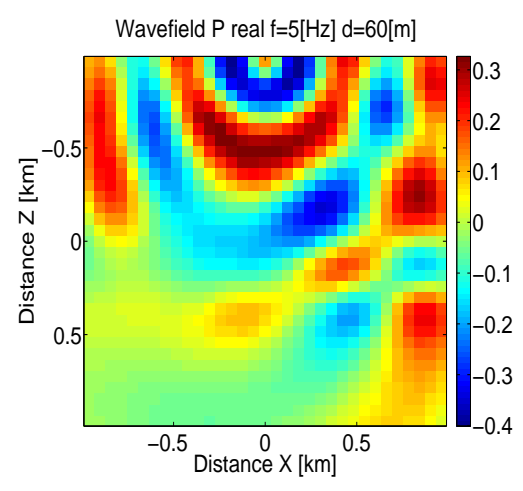

Figure 6: P-wave $5[\mathrm{~Hz}]$

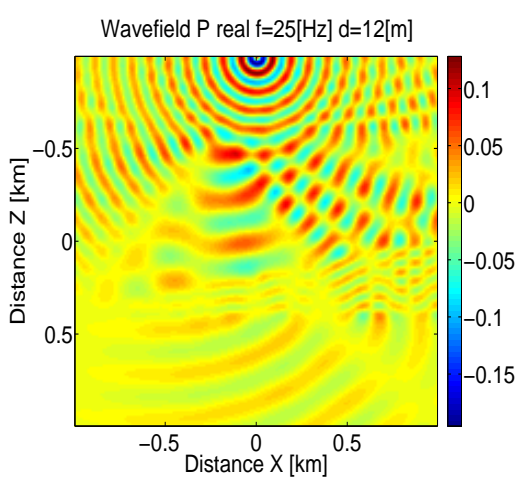

Figure 7: P-wave $25 \mathrm{~Hz}$

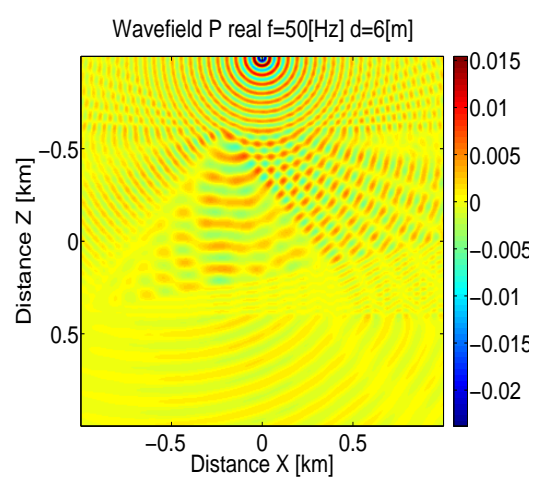

Figure 8: P-wave $50 \mathrm{~Hz}$ the method for different well known attenuation-dispersion models (Kolsky, Cole-Cole, General, [15]), and in particular noticed the clear difference between the attenuation of the amplitude of the wavefront due to the distribution of energy in the wavefront and the extra attenuation introduced by the damping functions.

This kind of behaviour has a notorious importance for methods like FWI. In the case of field exploration, subsurface information can be obtained through the use of Full Waveform Inversion. The quality of the information acquired through the inversion is off course dependent of the ability of the model to approach the physical properties of the medium.

\section{Acknowledgment}

This work is supported by Colombian Oil Company ECOPETROL and COLCIENCIAS as a part of the research project grant No. 0266-2013. JCMC wants to thanks to "Estrategia de sostenibilidad 2014-2015, Universidad de Antioquia".

\section{References}

[1] Carcione, J. M., D. Kosloff, \& R. Kosloff, 1988. "Wave propagation simulation in a linear viscoacoustic medium", Geophysics, 93, 393-407.

[2] Chen, J.-B., 2014. "Laplace-fourier-domain dispersion analysis of an average derivative optimal scheme for scalar-wave equation", Geophys. J Int., 197, 16811692.

[3] Deraemaeker A., Babuska I. \& Bouillard, P., 1999, "Dispersion and pollution of the fem solution for helmholtz equation in one, two and three dimensions", International Journal for Numerical Methods in Engineering, 46, 471-499.

[4] Fichtner, A., 2011, Full seismic waveform modelling and inversion, Springer.
[5] Huazhong, W., Libin, Z. \& Zaitian, MA., 2003, Seismic wave imaging in visco-acoustic media, Science in China Ser. A Mathematics. 47 146-154

[6] Jo, C.-H., 1996, "An optimal 9-point, finite-difference, frequency-space, 2-d scalar wave extrapolator", Geophysics, 61, 529-537.

[7] Kamei, R., \& R. G. Pratt, 2013, "Inversion strategies for visco-acoustic waveform inversion", Geophys. J Int., doi 10.1093/gij/ggt109.

[8] Lavergne, M., 1986, Seismic methods, Editions Technip.

[9] Marfurt, K., 1984, "Accuracy of finite-difference and finite-element modelling of the scalar and elastic wave equations", Geophysics, 49, 533-549.

[10] Mase, G., and G. E. Mase, 1999. Continuum mechanics for engineers, CRC Press.

[11] Mavko, G., T. Mukerji, and J. Dvorkin, 2009, The rock physics handbook, Cambridge University Press.

[12] Operto, S., \& J. Virieux, 2006, Practical aspects of frecuecny-domain finite-difference modelling of seismic wave propagation, Ecole thématique SEISCOPE.

[13] Shin, C. and Ho Cha, Y., 2008, "Waveform inversion in the Laplace domain", Geophys. J Int., 173, 922-931.

[14] Strikwerda, J., 2004, Finite difference schemes and partial differential equations, SIAM.

[15] Yanghua, W., 2008, Seismic inverse $Q$ filtering, Blackwell Publishing.

[16] Zongqing Yang, Liu, Y., \& Ren, Z. 2014, "Comparisons of visco-acoustic wave equations", Journal of Geophysics and Engineering,11,025004. 